\title{
Lattice-coupled Si/MXene confined by hard carbon for fast sodium-ion conduction
}

LiGou ${ }^{a}$, Weifeng Jing ${ }^{a}, Y_{i n g} \mathrm{Li}^{a}$, Mei Wang ${ }^{a}$, Shengliang Hu ${ }^{a}$, Huiqi Wang ${ }^{a *}$, Yan-Bing He

a School of Energy and Power Engineering \& School of Materials Science and Engineering, North University of China, Taiyuan 030051, P. R. China

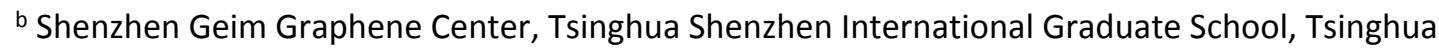
University, Shenzhen, 518055, P. R. China

*Corresponding author: hqiwang@nuc.edu.cn (H. Wang) 

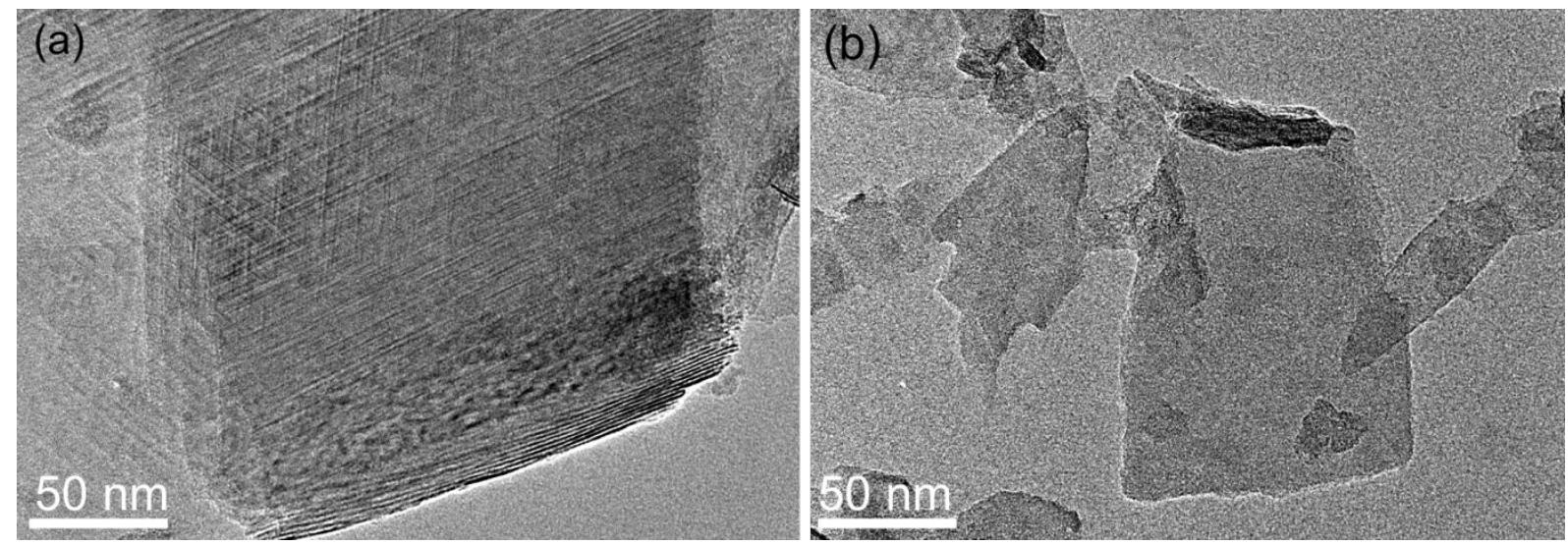

Figure S1 TEM images of (a) multilayer $d$-MXene and (b) monolayer $d$-MXene.

Table S1 Components (at. \%) of various element species in as-prepared materials.

\begin{tabular}{lccccc}
\hline \multicolumn{1}{c}{ No. } & C 1s & O 1s & Si 2p & Ti 2p & Others \\
\hline Pure Si & 11.42 & 33.60 & 54.98 & - & - \\
$d$-MXene & 17.75 & 35.54 & - & 15.95 & 31.76 \\
(Si/d-MXene)@HC & 33.15 & 30.57 & 20.00 & 6.05 & 10.23 \\
\hline
\end{tabular}

Table S2 The ratio values of $I_{D} / I_{G}$, the Raman shift of D-band and G-band.

\begin{tabular}{lccc}
\hline No. & $\begin{array}{c}\text { D-band } \\
\left(\mathrm{cm}^{-1}\right)\end{array}$ & $\begin{array}{c}\text { G-band } \\
\left(\mathrm{cm}^{-1}\right)\end{array}$ & $\begin{array}{c}\mathrm{I}_{\mathrm{D}} / \mathrm{I}_{\mathrm{G}} \\
(\text { Area })\end{array}$ \\
\hline$d$-MXene & 1363 & 1564 & 1.25 \\
(Si/d-MXene)@HC & 1377 & 1598 & 1.33 \\
\hline
\end{tabular}




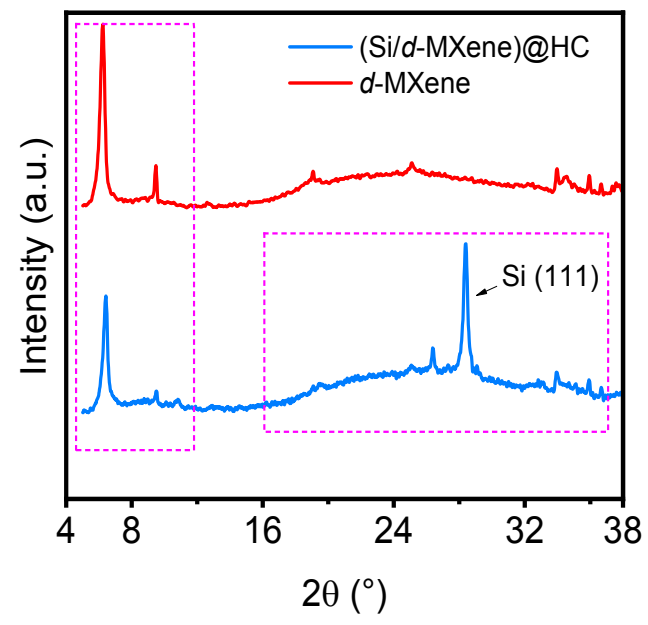

Figure S2 the enlarged XRD pattern of (Si/d-MXene)@HC and $d$-MXene.

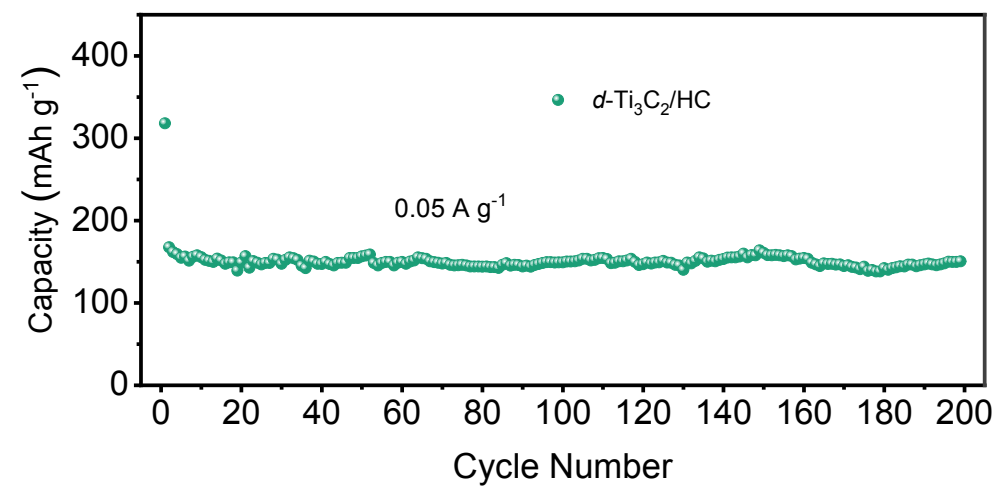

Figure S3 Cycling performance of $d$-MXene/HC electrode at a current density of $0.05 \mathrm{~A} \mathrm{~g}^{-1}$.

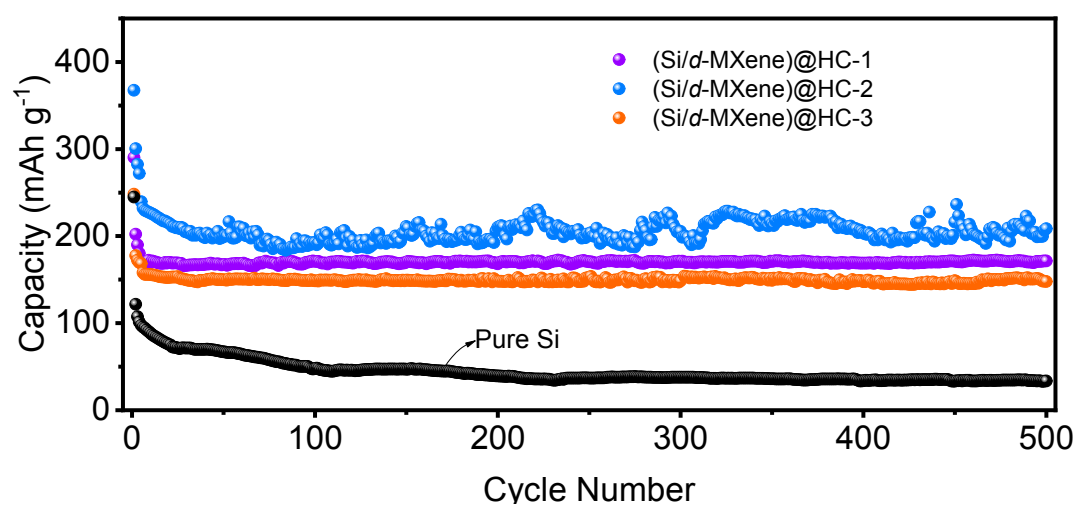

Figure S4 Cycling performance of (Si/d-MXene)@HC-1, (Si/d-MXene)@HC-2, (Si/dMXene)@HC-3 and pure Si electrodes at a current density of $0.05 \mathrm{~A} \mathrm{~g}^{-1}$. 
Table S3 Cycling performance of as-collected materials.

\begin{tabular}{lcccc}
\hline No. & $\begin{array}{c}\text { Si concentration } \\
(\text { wt. \%) }\end{array}$ & $\begin{array}{c}\text { Inversible Capacity } \\
\left(\text { mAh g- }^{-1}\right)\end{array}$ & $\begin{array}{c}\text { ICE } \\
(\%)\end{array}$ & $\begin{array}{c}\text { Capacity after 500 cycles } \\
\left(\text { mAh g }^{-1}\right)\end{array}$ \\
\hline (Si/d-MXene)@HC-1 & 7.9 & 202.2 & 71.3 & 171.4 \\
(Si/d-MXene)@HC-2 & 18.5 & 300.5 & 61.8 & 208.5 \\
(Si/d-MXene)@HC-3 & 37.4 & 177.5 & 50.6 & 147.8 \\
d-MXene & - & 165.1 & 47.3 & 125.6 \\
MXene & - & 145.5 & 45.5 & 108.3 \\
Pure Si & - & 29.8 & 35.3 & 9.6 \\
\hline
\end{tabular}

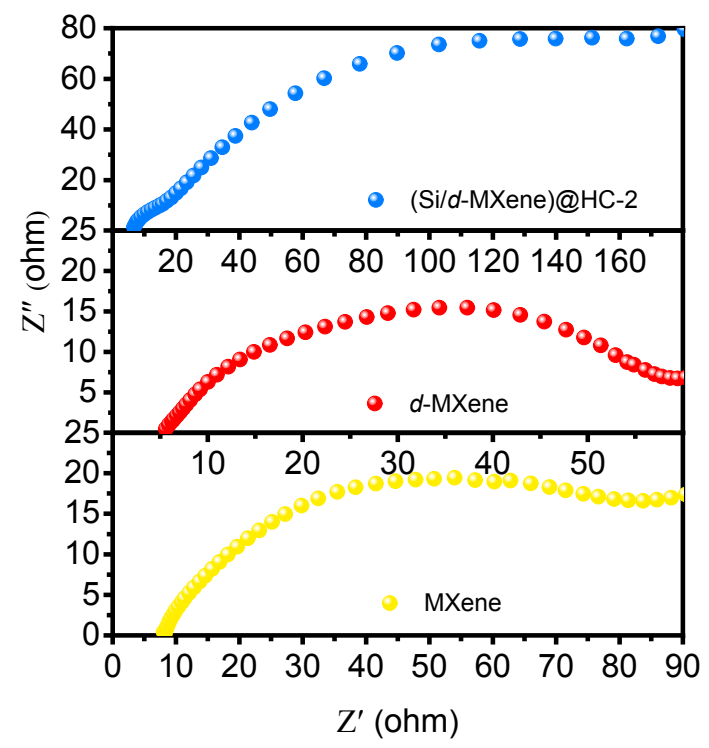

Figure S5 The enlarged EIS profile of (Si/d-MXene)@HC-2, $d$-MXene and MXene.

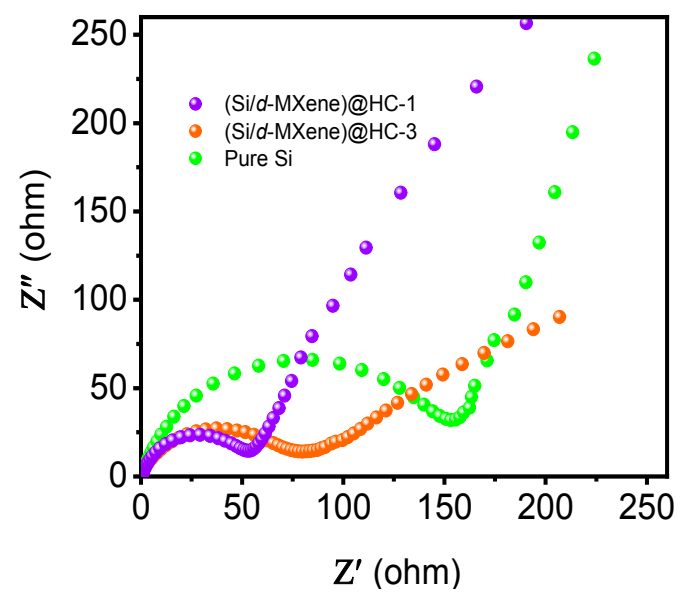

Figure S6 EIS profiles of different (Si/d-MXene)@HC electrodes and pure Si electrode. 


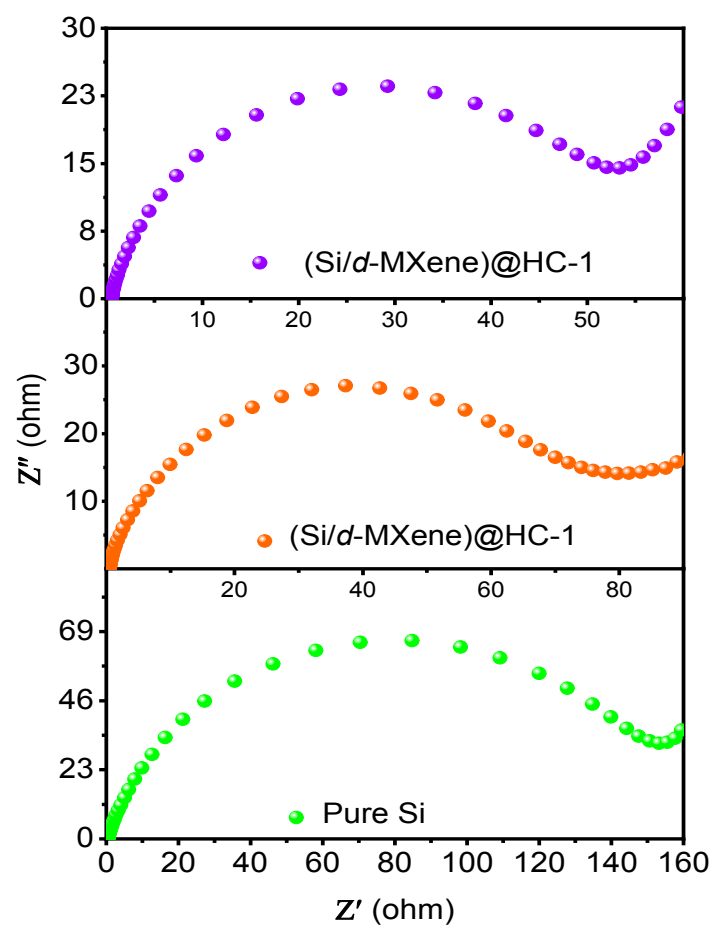

Figure S7 The enlarged EIS profiles of different (Si/d-MXene)@HC and pure Si electrodes. 
Table S4 The comparison of the Si-based composites for SIBs anode.

\begin{tabular}{|c|c|c|}
\hline Material/electrode & Cycling/rate performance (mAh $\mathrm{g}^{-1}$ ) & Year [References] \\
\hline Silicon oxycarbides & 160 at $0.025 \mathrm{~A} \mathrm{~g}^{-1}$ after 140 cycles & $2021[1]$ \\
\hline Biomass C-Si nanocomposites & 122 at $0.2 \mathrm{~A} \mathrm{~g}^{-1}$ after 100 cycles & $2020[2]$ \\
\hline Sponge-like amorphous silicon & 176 at $0.05 \mathrm{~A} \mathrm{~g}^{-1}$ after 100 cycles & $2018[3]$ \\
\hline Si/C nanocomposite & 280 at $0.1 \mathrm{~A} \mathrm{~g}^{-1}$ after 100 cycles & $2016[4]$ \\
\hline 3DHCN@Si-60 composite & 105 at $0.1 \mathrm{~A} \mathrm{~g}^{-1}$ after 1000 cycles & $2019[5]$ \\
\hline $\mathrm{Si} / \mathrm{SiO}_{2}-\mathrm{OMC}$ composite & 190 at $.1 .0 \mathrm{~A} \mathrm{~g}^{-1}$ after 100 cycles & $2018[6]$ \\
\hline Amorphous Silicon & 150 at $0.04 \mathrm{~A} \mathrm{~g}^{-1}$ after 100 cycles & \multirow{2}{*}{$2016[7]$} \\
\hline Sn coated Si & 140 at $0.05 C$ after 5 cycles & \\
\hline Silicon nanowires & 125 at $0.1 \mathrm{C}$ after 100 cycles & $2018[8]$ \\
\hline Silicon-Graphene film & 120 at $0.1 C$ after 100 cycles & 2017 [9] \\
\hline Nanoscaled Si & 65 at $0.05 \mathrm{~A} \mathrm{~g}^{-1}$ after 100 cycles & \multirow{2}{*}{$2017[10]$} \\
\hline Si/C fiber nanocomposite & 298 at $0.05 \mathrm{~A} \mathrm{~g}^{-1}$ after 300 cycles & \\
\hline 3D Si/Ge nanorods array & 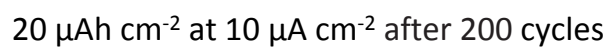 & $2015[11]$ \\
\hline Si/(d-MXene)-HC & 208 at $0.05 \mathrm{~A} \mathrm{~g}^{-1}$ after 500 cycles & This Work \\
\hline
\end{tabular}

Table S5 Kinetic parameters calculated by fitting an equivalent circuit.

\begin{tabular}{lccc}
\hline Sample & $\mathrm{R}_{\mathrm{e}}$ (ohm) & $\mathrm{R}_{\mathrm{SEI}}$ (ohm) & $\mathrm{R}_{\mathrm{ct}}$ (ohm) \\
\hline (Si/d-MXene)@HC-1 & 4.9 & 11.0 & 50.5 \\
(Si/d-MXene)@HC-2 & 6.7 & 8.1 & 75.7 \\
(Si/d-MXene)@HC-3 & 4.8 & 12.8 & 77.9 \\
d-MXene & 5.6 & 10.9 & 42.2 \\
MXene & 8.3 & 6.3 & 66.8 \\
Pure Si & 4.5 & 28.9 & 149.8 \\
\hline
\end{tabular}



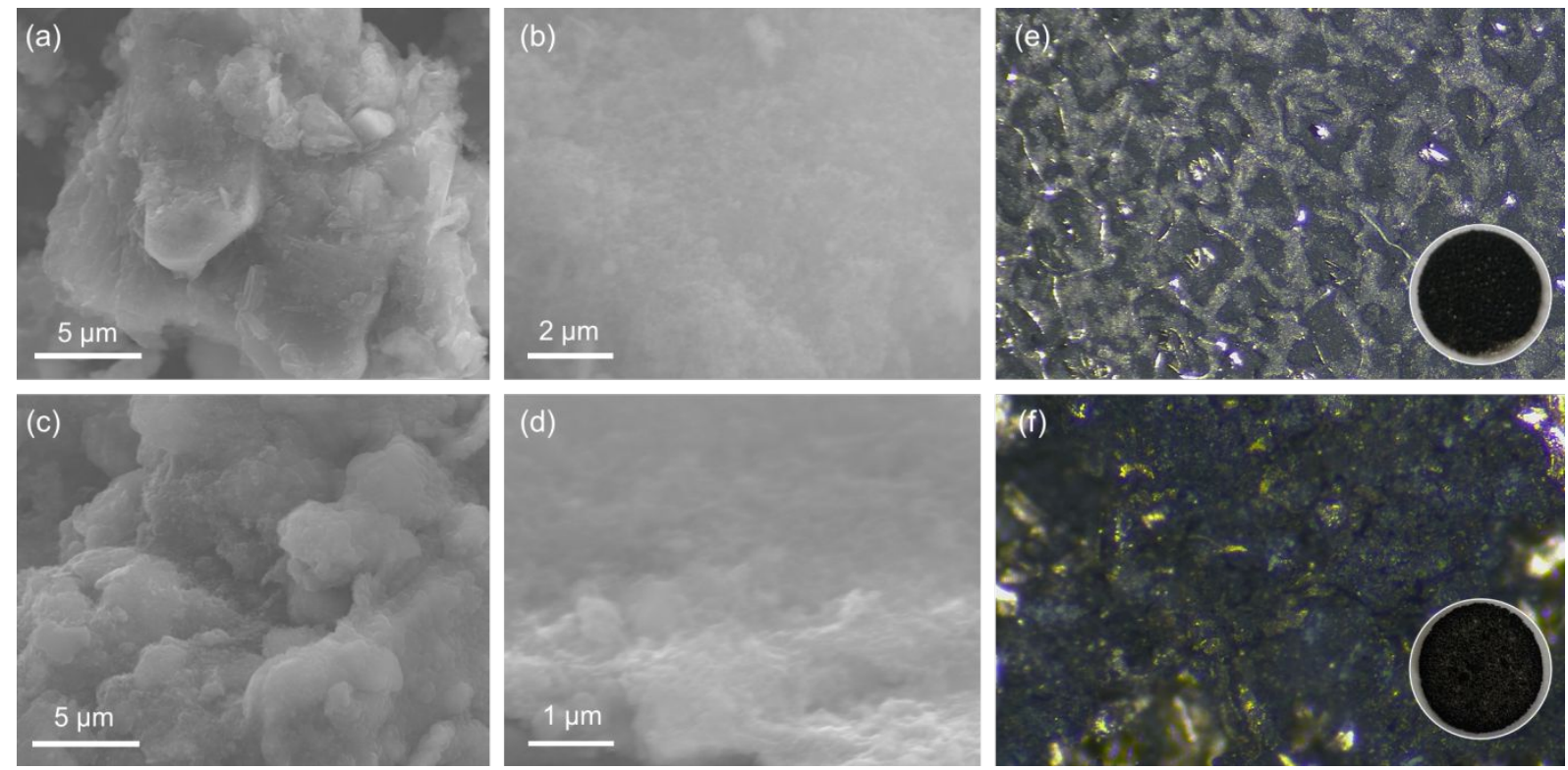

Figure S8 SEM images with different magnifications of the (Si/d-MXene)@HC electrode before cycle $(a, b)$ and after 500 cycles (c, d), and the optical photographs of the (Si/d-MXene)@HC electrode before cycle (e) and after 500 cycles (f).

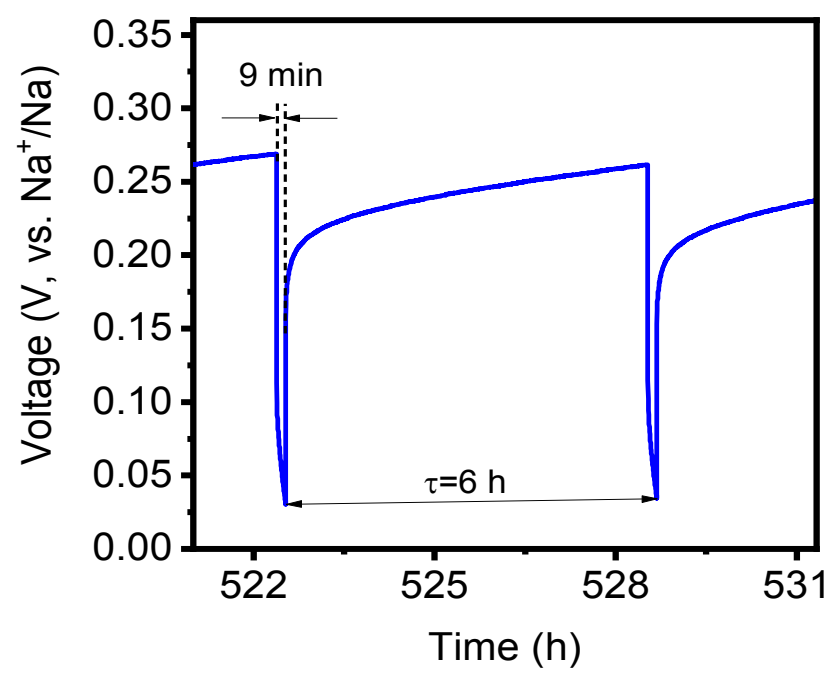

Figure \$9 The voltage profile of a GITT unit of (Si/d-MXene)@HC electrode 
Detailed analysis of GITT curve as follows:

The Fick second law with the following equation (1) was applied to measure the ionic diffusion coefficient. $^{12,13}$

$\mathrm{D}=\frac{4}{\pi}\left(\frac{m_{b} V_{M}}{M_{B} S}\right)^{2}\left(\frac{\Delta E_{S}}{\tau(d E / d \sqrt{\tau})}\right)^{2} \quad\left(\tau \ll \mathrm{L}^{2} / \mathrm{D}\right) \quad$ (1) 14,15

Based on the linear relationship between cell voltage and $\tau^{1 / 2}$, Equation (1) can be further changed into equation (2)

$\mathrm{D}=\frac{4}{\pi \tau}\left(\frac{m_{b} V_{M}}{M_{B} S}\right)^{2}\left(\frac{\Delta E_{S}}{\Delta E_{\tau}}\right)^{2} \quad\left(\tau \ll \mathrm{L}^{2} / \mathrm{D}\right) \quad$ (2) 14,15

where $D$ is the $\mathrm{Na}^{+}$diffusion coefficient, $\tau$ is the duration of impulse current, $n_{B}$ and $V_{M}$ is the amount of substance and molar volume of the active substance, $S$ is the area of the electrode. $\Delta E_{S}$ is the two adjacent stable potential difference, $\Delta E_{\tau}$ is the potential change caused by impulse current. Galvanostatic intermittent titration technique (GITT) technology was used to study the intrinsic dynamic characteristics of the (Si/d-MXene)@HC electrode in the discharge/charge process.

\section{References}

1. Chandra, C.; Devina, W.; Alvin, S.; Kim, J., New strategy for increasing sodium-ion uptake in silicon oxycarbides. Chem Eng J 2021, 404, 126520.

2. Sekar, S.; Ahmed, A. T. A.; Kim, D. Y.; Lee, S., One-Pot Synthesized Biomass C-Si Nanocomposites as an Anodic Material for High-Performance Sodium-Ion Battery. Nanomaterials 2020, 10 (9), 1728.

3. Han, Y.; Lin, N.; Xu, T.; Li, T.; Tian, J.; Zhu, Y.; Qian, Y., An amorphous Si material with a sponge-like structure as an anode for Li-ion and Na-ion batteries. Nanoscale 2018, 10 (7), 3153-3158.

4. Zhao, Q.; Huang, Y.; Hu, X., A Si/C nanocomposite anode by ball milling for highly reversible sodium storage. Electrochemistry Communications 2016, 70, 8-12.

5. Wu, J.; Xu, G.; Liu, X.; Li, Z.; Yang, L.; Chu, P. K., Self-supported electrodes composed of silicon nanocrystals in 3D hierarchical carbon network for reversible sodium storage. J Mater Sci: Mater Electron 2019, $30(3), 2732-2742$. 
6. Zeng, L.; Liu, R.; Han, L.; Luo, F.; Chen, X.; Wang, J.; Qian, Q.; Chen, Q.; Wei, M., Preparation of a Si/SiO ${ }_{2}-$ Ordered-Mesoporous-Carbon Nanocomposite as an Anode for High-Performance Lithium-Ion and Sodium-Ion Batteries. Chemistry - A European Journal 2018, 24 (19), 4841-4848.

7. Lim, C.-H.; Huang, T.-Y.; Shao, P.-S.; Chien, J.-H.; Weng, Y.-T.; Huang, H.-F.; Hwang, B. J.; Wu, N.-L., Experimental Study on Sodiation of Amorphous Silicon for Use as Sodium-Ion Battery Anode. Electrochim Acta 2016, $211,265-272$.

8. Jangid, M. K.; Lakhnot, A. S.; Vemulapally, A.; Sonia, F. J.; Sinha, S.; Dusane, R. O.; Mukhopadhyay, A., Crystalline core/amorphous shell structured silicon nanowires offer size and structure dependent reversible Nastorage. J Mater Chem A 2018, 6 (8), 3422-3434.

9. Jangid, M. K.; Vemulapally, A.; Sonia, F. J.; Aslam, M.; Mukhopadhyay, A., Feasibility of Reversible Electrochemical Na-Storage and Cyclic Stability of Amorphous Silicon and Silicon-Graphene Film Electrodes. $J$ Electrochem Soc 2017, 164 (12), A2559-A2565.

10. Zhang, L.; Hu, X.; Chen, C.; Guo, H.; Liu, X.; Xu, G.; Zhong, H.; Cheng, S.; Wu, P.; Meng, J.; Huang, Y.; Dou, S.; Liu, H., In Operando Mechanism Analysis on Nanocrystalline Silicon Anode Material for Reversible and Ultrafast Sodium Storage. Adv Mater 2017, 29 (5), 1604708.

11. Yue, C.; Yu, Y.; Sun, S.; He, X.; Chen, B.; Lin, W.; Xu, B.; Zheng, M.; Wu, S.; Li, J.; Kang, J.; Lin, L., High Performance 3D Si/Ge Nanorods Array Anode Buffered by TiN/Ti Interlayer for Sodium-Ion Batteries. 2015, 25 (9), 1386-1392.

12. Ding, N.; Xu, J.; Yao, Y. X.; Wegner, G.; Fang, X.; Chen, C. H.; Lieberwirth, I., Determination of the diffusion coefficient of lithium ions in nano-Si. Solid State Ionics 2009, 180 (2), 222-225.

13. Pan, K.; Zou, F.; Canova, M.; Zhu, Y.; Kim, J.-H., Systematic electrochemical characterizations of Si and $\mathrm{SiO}$ anodes for high-capacity Li-Ion batteries. Journal of Power Sources 2019, 413, 20-28.

14. Wang, H.; An, D.; Li, N.; Li, Y.; Wang, M.; Zhang, J.; Hu, S.; He, Y.-B., PbTe nanodots confined on ternary $\mathrm{B}_{2} \mathrm{O}_{3} / \mathrm{BC}_{2} \mathrm{O} / \mathrm{C}$ nanosheets as electrode for efficient sodium storage. Journal of Power Sources 2020, 461, 228110.

15. Wang, H.; Gou, L.; Jing, W.; An, D.; Li, Y.; Wang, M.; Li, N.; Hu, S.; He, Y.-B., Highly microporous $\mathrm{SbPO}_{4} / \mathrm{BC}_{\mathrm{x}}$ hybrid anodes for sodium-ion batteries. Materials Advances 2020, 1 (2), 206-214. 